Pacific Journal of Mathematics

FINITELY GENERATED MODULES OVER BEZOUT RINGS 


\section{FINITELY GENERATED MODULES OVER BEZOUT RINGS}

\section{Roger Wiegand and Sylvia Wiegand}

Let $R$ be a Bezout ring (a commutative ring in which all finitely generated ideals are principal), and let $M$ be a finitely generated $\boldsymbol{R}$-module. We will study questions of the following sort: (A) If every localization of $M$ can be generated by $n$ elements, can $M$ itself be generated by $n$ elements? (B) If $M \oplus R^{m} \cong R^{n}$ for some $m, n$, is $M$ necessarily free? (C) If every localization of $M$ has an element with zero annihilator, does $M$ itself have such an element? We will answer these and related questions for various familiar classes of Bezout rings. For example, the answer to $(B)$ is "no" for general Bezout rings but "yes" for Hermite rings (defined below). Also, a Hermite ring is an elementary divisor ring if and only if (A) has an affirmative answer for every module $M$.

1. Preliminaries. All rings in this discussion are commutative with 1 , and modules are unital. If $A$ is an $m \times n$ matrix over $R$, let $\operatorname{Ker}(A)$ be the set of columns $X \in R^{n}$ for which $A X=0$ and let $\operatorname{Im}(A)$ be the submodule of $R^{m}$ generated by the columns of $A$. The $k \times k$ identity matrix is denoted by $I_{k}$, and $A^{T}$ is the transpose of $A$. We say the $R$-module $M$ is "named" by the matrix $A$ if $M \cong \operatorname{Coker}(A)=$ $R^{m} / \operatorname{Im}(A)$.

We define the Fitting invariants $F_{i}(A)$ of an $m \times n$ matrix $A$ as follows: $F_{i}(A)$ is the ideal of $R$ generated by the $(m+1-i) \times$ $(m+1-i)$ minors of $A$, for $0 \leqq i \leqq m$ and $F_{i}(A)=R$ for $i>m$. If $A$ names the module $M$, we put $F_{i}(M)=F_{i}(A)$. (For a proof that this is unambiguous, consult [6, p. 146] for example.) The following wellknown lemma will be useful later:

LeMma 1.1. Let $M$ be any finitely presented module. then $F_{1}(M) \subseteq(0: M)$. If $M$ is free of rank $r$, then $F_{r}(M)=0$ and $F_{r+1}(M)=R$.

For $M$ a finitely generated $R$-module, we denote by $\mu(R, M)$ the minimum number of generators required for $M$ over $R$.

Proposition 1.2. A commutative ring $R$ is Bezout if and only if every finitely presented $R$-module can be named by a square matrix.

Proof. For the "if" implication, let $a, b \in R$ and let $M=$ $R /(a, b)$. Let $A$ be a square matrix that names $M$. Then $(a, b)=$ 
$F_{1}(M)=(\operatorname{det} A)$, a principal ideal. The "only if" implication is a consequence of the next lemma, communicated to us by Lawrence Levy.

LEMMA 1.3. Let $R$ be a ring in which $\mu(R, I) \leqq k$ for every finitely generated ideal $I$. Let $M$ and $N$ be finitely generated $R$-modules, with $M \subseteq N$. Then $\mu(R, M) \leqq k \cdot \mu(R, N)$.

Proof. It is easy to see that we may assume $N=R^{n}$. For $M \subseteq R^{n}$, we show that $\mu(R, M) \leqq k n$, by induction on $n$, the case $n=1$ being true by hypothesis. Suppose $\left\{m_{\alpha}\right\}$ is a finite set of generators for $M$ and set $m_{\alpha}=\left(u_{\alpha}, r_{\alpha}\right)$, where $u_{\alpha} \in R^{n-1}, r_{\alpha} \in R$. Let $\pi$ be the projection from $R^{n}$ onto its first $n-1$ coordinates; then by induction $\pi(M)$ can be generated by $k(n-1)=t$ elements $v_{1}, v_{2}, \cdots, v_{t}$. Choose $s_{j} \in R$ so that $\left(v_{j}, s_{j}\right) \in M$ and choose $a_{\alpha j}$ so that $u_{\alpha}=\Sigma_{j} a_{\alpha j} v_{j}$. Then $m_{\alpha}=\Sigma_{j} a_{\alpha j}\left(v_{j}, s_{j}\right)+\left(0, r_{\alpha}-\Sigma_{j} a_{\alpha j} s_{j}\right)$. The ideal generated by the $r_{\alpha}-$ $\sum_{j} a_{\alpha j} s_{j}$ may be generated by $k$ elements $b_{1}, b_{2}, \cdots, b_{k}$ of $R$. Clearly, the $n k$ elements $\left(v_{1}, s_{1}\right), \cdots,\left(v_{t}, s_{t}\right),\left(0, b_{1}\right), \cdots,\left(0, b_{k}\right)$ generate $M$.

We remark that the proof of (1.3) works perfectly well in the noncommutative case, thus answering a question of Pierce $[8, \$ 25,(1)]$.

A projective module $M$ is said to have (constant) rank $n$ provided $\mu\left(R_{P}, M_{P}\right)=n$ for every $P$. It is known [11] that every projective of constant rank is finitely generated. The following lemma (see, for example, the proof of [11, Proposition 1.4]) essentially reduces the study of finitely generated projectives to the case of constant rank:

LEMMA 1.4. For every finitely generated projective module $M$ there exist orthogonal idempotents $e_{0}, \cdots, e_{n}$ with sum 1 , such that $M e_{k}$ has constant rank $k$ as an $R_{k}$-module for all $k$.

It is easy to see that $\operatorname{Re}_{0}\left(e_{0}\right.$ as above) is the annihilator of $M$. In particular, $M$ is faithful if and only if $e_{0}=0$, that is, if and only if $\mu\left(R_{P}, M_{P}\right) \neq 0$ for all $P$. Recall that an element $m \in M$ is unimodular provided $R m$ is a nonzero, free direct summand of $M$. The following result is a typical application of (1.4). The proof will be omitted.

Proposition 1.5. These conditions on a ring are equivalent: (i) Every finitely generated, faithful projective module has a unimodular element. (ii) Every projective module of constant rank is free. (iii) Every finitely generated projective module is a direct sum of cyclics.

A ring $R$ is Hermite if every matrix over $R$ is equivalent to an upper triangular matrix. If every matrix is equivalent to a diagonal 
matrix $\left(a_{i j}\right)$ in which $a_{i i}$ divides $a_{i+1, i+1}, R$ is an elementary divisor ring. It has recently been shown [7] that a ring is an elementary divisor ring if and only if every finitely presented module is a direct sum of cyclics. We will see later, in (3.4), that the conditions of (1.5) also have a matrix-theoretic formulation. We will need the following wellknown characterization of projective modules in terms of Fitting invariants:

Proposition 1.6. Let $M$ be a finitely presented $R$-module. Then $M$ is a rank $r$ projective module if and only if $F_{r}(M)=0$ and $F_{r+1}(M)=$ (1). More generally, $M$ is projective if and only if each $F_{k}(M)$ is generated by an idempotent.

2. The main theorem. If $M$ is a finitely generated module over a principal ideal ring or a von Neumann regular ring [8, Corollary 13.8], then $\mu(R, M)=\max \mu\left(R_{P}, M_{P}\right), P$ ranging over all maximal ideals. It seems reasonable to ask which Bezout rings have this property. For Hermite rings, we have a complete answer:

THEOREM 2.1. The following conditions on a Hermite ring $R$ are equivalent: (i) $\mu(R, M)=\max _{P} \mu\left(R_{P}, M_{P}\right)$, for every finitely generated $R$-module $M$. (ii) Every finitely presented, locally cyclic $R$-module is cyclic. (iii) For each $d \in R$, every projective $R /(d)$-module of rank 1 is free. (iv) For each ideal $I \subseteq R$, every projective $R / I$-module of constant rank is free. (v) $R$ is an elementary divisor ring.

Proof. Trivially, (i) implies (ii), and (iv) implies (iii). Since an $\boldsymbol{n}$-generator projective of constant rank $\boldsymbol{n}$ is clearly free, we see that (i) implies (iv), and (ii) implies (iii). Therefore it will suffice to show that (iii) implies (v) and (v) implies (i).

Assume (iii) holds. In order to prove that $R$ is an elementary divisor ring it will suffice, by the proof of [7, Theorem 3.8], to show that if $(a, b, c)=(1)$ then the module $M$ named by the matrix $A=\left[\begin{array}{ll}a & b \\ 0 & c\end{array}\right]$ is cyclic. Set $d=a c$. By (1.1) and (1.6), $M$ is a rank 1 projective $R /(d)$-module; then (iii) implies that $M$ is cyclic.

Finally we show that (v) implies (i). Suppose, first of all, that $M$ is finitely presented. Then by (1.2), $M$ is named by an $n \times n$ diagonal matrix $\left(d_{i j}\right)$ in which $\left(d_{i i}\right) \subseteq\left(d_{i+1, i+1}\right)$ for all $i$. Thus $M \cong$ $R /\left(d_{11}\right) \oplus \cdots \oplus R /\left(d_{m m}\right)$, where $m \leqq n$ and $\left(d_{m m}\right) \neq R$. Choosing a maximal ideal $P$ containing $d_{m m}$, we have $\mu(R, M)=\mu\left(R_{P}, M_{P}\right)=m$, as desired. For the general case, we can appeal to the following lemma: 
LEMMA 2.2. Let $R$ be any commutative ring, and let $M$ be a finitely generated $R$-module such that $\mu\left(R_{P}, M_{P}\right) \leqq n$ for each maximal ideal $P$. Then there exists a finitely presented module $N$, with $\mu\left(R_{P}, N_{P}\right) \leqq n$ for all $P$, and a surfection $N \rightarrow \rightarrow M$.

Proof. Choose a surjection $\phi: R^{k} \rightarrow \rightarrow M$ with kernel $K$, and let $P$ be an arbitrary prime ideal of $R$. By hypothesis, there are elements $u_{1}, \cdots, u_{n} \in R^{k}$ such that the images of $\phi\left(u_{i}\right)$ generate $M_{P}$. Then there are elements $v_{1}, \cdots, v_{k} \in K$ so that the images in $R_{P}^{k}$ of $v_{1}, \cdots, v_{k}, u_{1}, \cdots, u_{n}$ generate $R_{P \text {. }}^{k} \quad$ Let $K(P)$ be the sub-module of $K$ generated by the $v_{i}$, and set $N(P)=R^{k} / K(P)$. Then $N(P)$ is finitely presented, and $\mu\left(R_{P}, N(P)_{P}\right) \leqq n$. By [2, Hilfsatz 2] there is a neighborhood $U(P)$ of $P$ (in the Zariski topology on $\operatorname{spec}(R)$ ) such that $\mu\left(R_{Q}, N(P)_{Q}\right) \leqq n$ for all $Q \in U(P)$. Now administer the same treatment to every prime $P$, let $U\left(P_{1}\right), \cdots, U\left(P_{n}\right) \operatorname{cover} \operatorname{spec}(R)$, and set $N=R^{n} /\left(K\left(P_{1}\right)+\cdots+K\left(P_{n}\right)\right)$.

We remark that there may be primes $P$ for which $\mu\left(R_{P}, N_{P}\right)>$ $\mu\left(R_{P}, M_{P}\right)$, even though the same bound $n$ suffices. In fact, the following proposition tells exactly when $N$ can be chosen so that $\mu\left(R_{P}, N_{P}\right)=\mu\left(R_{P}, M_{P}\right)$ for all $P$.

PROPOSITION 2.3. The following conditions on $R$ are equivalent : (i) For each finitely generated $M$ there exist a finitely presented $N$, with $\mu\left(R_{P}, N_{P}\right)=\mu\left(R_{P}, M_{P}\right)$ for all $P$, and a surjection $N \rightarrow \rightarrow M$. (ii) For each cyclic $R$-module $C$ there is a finitely presented $N$, with $\mu\left(R_{P}, N_{P}\right)=$ $\mu\left(R_{P}, C_{P}\right)$ for all $P$. (iii) $R$ has Noetherian prime spectrum.

Proof. Certainly (i) implies (ii). To show that (ii) implies (iii) we have to show that every open set $U$ is quasi-compact. Now $U=$ $\{P \in \operatorname{spec}(R) \mid P \nsupseteq I\}$ for some ideal $I$. Let $C=R / I$ and choose $N$ as in (ii). Then $U=\left\{P \in \operatorname{spec}(R) \mid N_{P}=0\right\}=\left\{P \in \operatorname{spec}(R) \mid P \nsupseteq F_{1}(N)\right\}$, by (1.1), and the latter set is quasi-compact since $F_{1}(N)$ is finitely generated. Finally, to prove (iii) implies (i), let $M$ be any finitely generated module, and set $V_{n}=\left\{P \in \operatorname{spec}(R) \mid \mu\left(R_{P}, M_{P}\right) \leqq n\right\}$. Then each $V_{n}$ is quasi-compact (since $\operatorname{spec}(R)$ is Noetherian) and open in $\operatorname{spec}(R)$, by [2, Hilfsatz 2]. It follows that the sets $U_{n}=$ $\left\{P \in \operatorname{spec}(R) \mid \mu\left(R_{P}, M_{P}\right)=n\right\}$ are open and closed in the patch topology on $\operatorname{spec}(R),[12]$. As in the proof of (2.2), choose a surjection $\phi: R^{k} \rightarrow \rightarrow M$, and fix a prime $P$, say $P \in U_{n}$. Choose $u_{i}, v_{j}$ as in the proof of (2.2) and define $K(P), N(P)$ and $U(P)$ as before. The proof is completed by replacing $U(P)$ by $U(P) \cap U_{n}$, and then using compactness of $\operatorname{spec}(R)$ relative to the patch topology. 
It is unknown whether the conditions of Theorem 2.1 are equivalent to the apparently weaker condition: Every projective of constant rank is free. If so, it would follow that every semihereditary Bezout ring is an elementary divisor ring. (Reason: Over any semi-hereditary ring, every projective module, finitely generated or not, is a direct sum of finitely generated ideals, [5].) In particular, this would answer the long-standing question, mentioned in [4] and [1, p. 290]: "Is every Bezout domain an elementary divisor ring?"

We remark that there are Hermite rings that are not elementary divisor rings [3]. Thus by passing to an appropriate homomorphic image, we obtain an example of a Hermite ring with a rank 1 projective that is not free.

We conclude this section with an example of an elementary divisor ring with a projective module (necessarily non-finitely generated), that is not a direct sum of cyclics. Let $\mathbf{R}^{+}$be the space of nonnegative real numbers, and let $R$ be the ring of continuous real-valued functions on $\beta \mathbf{R}^{+}-\mathbf{R}^{+}$, where $\beta$ is the Stone-Cech compactification. This space is connected $[3,2.8]$, so $R$ has no nontrivial idempotents. By $[3,3.3,4.1$, 4.2] $R$ is an elementary divisor ring. Obviously $R$ is not a domain, so we can apply:

Proposition 2.4. Let $R$ be a semiprime Bezout ring with no nontrivial idempotents. If every projective ideal of $R$ is a direct sum of principal ideals, then $R$ is a domain.

Proof. Since the annihilator of a projective principal ideal is generated by an idempotent, every projective ideal of $R$ is free. In particular, projective ideals are finitely generated. By [11], every finitely generated flat module is projective. Let $\boldsymbol{P}$ be any minimal prime of $R$. Since every localization of $R$ is a valuation domain, we see that $R / P$ is flat. Hence $P=0$ as desired.

3. Stably free modules. We turn now to a very special class of constant rank projectives, the stably free modules. Using (1.1) we see that a module $M$ satisfies $M \oplus R^{m} \cong R^{n}$ if and only if $M \cong \operatorname{ker} A$ for some $m \times n$ matrix $A$ with $F_{1}(A)=(1)$. We want a matrixtheoretic condition for a stably free module to be free. This criterion is well-known in case $m=1$. The proof of the general case is rather straightforward, and will be omitted.

Proposition 3.1. Let $A$ be an $m \times n$ matrix with $F_{1}(A)=$ (1). Then ker $A$ is free if and only if $A$ can be filled out to an $n \times n$ invertible matrix. 
Corollary 3.2. Over a Hermite ring every stably free projective module is free.

Proof. Let $A$ be an $m \times n$ matrix with $F_{1}(A)=R$. Choose invertible matrices $P, Q$ such that $P A Q$ is lower triangular. Now $m \leqq n$, so $P A Q=[B \mid 0]$ for an invertible $B$. Then $A Q=\left[P^{-1} B \mid 0\right]$ can certainly be filled out to an invertible $n \times n$ matrix $C$; and $C Q^{-1}$ has $A$ as its top $m$ rows.

EXAMPLE 3.3. A Bezout ring with a stably free projective that is not free.

For any topological space $Y$, let $C(Y)$ denote the ring of continuous real-valued functions on $Y$. Let $X$ be the disjoint union of countably many 2 -spheres. To be precise, let $X=\cup_{n} X_{n}$, where $X_{n}=$ $\left\{\left(a_{1}, a_{2}, a_{3}\right) \in \mathbf{R}^{3}\right.$ such that $\left.\left(a_{1}-3 n\right)^{2}+\left(a_{2}-3 n\right)^{2}+\left(a_{3}-3 n\right)^{2}=1\right\}$. For $i=1,2,3$, let $\phi_{i} \in C(X)$ be the (continuous) function defined by $\phi_{i}(x)=$ $a_{i}-3 n$, if $x=\left(a_{1}, a_{2}, a_{3},\right) \in X_{n}$. Each of these "coordinate" functions has a unique extension (still denoted by $\phi_{i}$ ) to the Stone-Cech compactification $\beta X$, and we have $\phi_{1}^{2}+\phi_{2}^{2}+\phi_{3}^{2}=1$. Set $R=$ $C(\beta X-X)$. Since $X$ is countably compact and locally compact, [3, Theorem 2.7] implies $R$ is Bezout. Let bars denote passage from $\dot{C}(\beta X)$ to $C(\beta X-X)$ via restriction. Note that $X$ is open in $\beta X$, so the map $f \rightarrow \bar{f}$ maps $C(\beta X)$ onto $R$.

Suppose $\left[\bar{\phi}_{1} \bar{\phi}_{2} \bar{\phi}_{3}\right]$ is the first row of an invertible matrix over $R$. Then there is a matrix $A$ over $C(\beta X)$ with first row $\left[\phi_{1} \phi_{2} \phi_{3}\right]$ such that $\bar{\delta}$ is a unit, where $\delta=\operatorname{det} A$. Now it is well-known that for each $n$, there is a point $x_{n} \in X_{n}$ for which $\delta\left(x_{n}\right)=0$. (To see this, let $\left[\alpha_{1}, \alpha_{2}, \alpha_{3}\right]$ be the second row of $A$. If $\delta(x) \neq 0$ for all $x \in X_{n}$, then the vector $\left(\alpha_{1}(x), \alpha_{2}(x), \alpha_{3}(x)\right)$ would be linearly independent of the vector $\left(\phi_{1}(x), \phi_{2}(x), \phi_{3}(x)\right)$ for each $x$. Since the latter vector is normal to the tangent plane of $X_{n}$ at $x$, the projection of $\left(\alpha_{1}(x), \alpha_{2}(x), \alpha_{3}(x)\right)$ would define a nonvanishing tangent vector field on $X_{n}$.) By compactness, the closure of the set $\left\{x_{n}\right\}$ in $\beta X$ must contain a point $p$ in $\beta X-X$. Then $\bar{\delta}(p)=\delta(p)=0$, contradicting the assumption that $\bar{\delta}$ is a unit.

As another application of (3.1) we give a matrix-theoretic characterization of those rings whose projectives of constant rank are free.

COROLlary 3.4. These conditions are equivalent for a ring $R$ : (i) Every projective module of constant rank is free. (ii) Every $m \times n$ matrix $A$ with $F_{r}(A)=0$ and $F_{r+1}(A)=(1)$ is equivalent to the $m \times n$ matrix with $I_{m-r}$ in the top left corner and 0's elsewhere. (iii) Every matrix with idempotent Fitting invariants is equivalent to a diagonal matrix. 
Proof. The only nontrivial assertion is (i) implies (ii). Suppose every projective module of constant rank is free, and let $A$ be an $m \times n$ matrix with $F_{r}(A)=0, F_{r+1}(A)=(1)$. Then $\left.\operatorname{Coker} A\right) \cong R^{r}, \operatorname{Im}(A) \cong$ $R^{m-r}$, and $\operatorname{Ker}(A) \cong R^{n-m+r}$. By filling in the following diagram

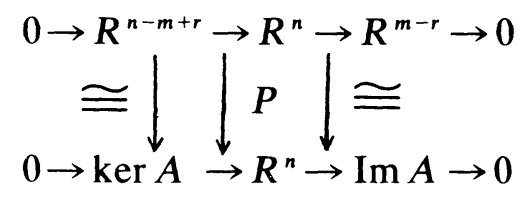

we obtain an invertible matrix $P$ such that $A P=[B \mid 0]$ for some $m \times(m-r)$ matrix $B$. Since $F_{1}\left(B^{T}\right)=(1), B$ can be filled out to an invertible $m \times m$ matrix $Q=[B \mid C]$, by (3.1). Then $Q^{-1} A P=\left[\begin{array}{rr}\mathrm{I}_{m-r} 0 \\ 0 & 0\end{array}\right]$, as desired.

4. Basic elements. Following Swan [9], we say an element $m$ in a finitely generated module $M$ is basic at a prime $P$, provided $\mu\left(R_{P},(M / R m)_{P}\right)<\mu\left(R_{P}, M_{P}\right)$. Equivalently, $m / 1$ is part of a minimal generating set for $M_{P}$. The element $m$ is said to be basic in $M$ provided it is basic at every prime $P$ of $R$. One can ask the following question, by analogy with the considerations of the preceding two sections: If $M$ is a finitely generated module over a Bezout ring and $M_{P} \neq 0$ for all $P$, does $M$ have a basic element? It is not hard to see that the answer is "yes" for Bezout domains. (In fact, in this case $M$ will have a unimodular element.) In general, however, the answer is "no", even for Boolean rings!

For a subset $A$ of the ring $R$ we let $D(A)$ be the (open) subset of $\operatorname{spec}(R)$ consisting of those primes not containing $A$. If $\alpha$ is an element of $R$ (or of an $R$-module $M$ ) we let $\alpha(P)$ denote the canonical image of $\alpha$ in $R_{P}$ (or in $M_{P}$ ). Let $Z(\alpha)=\{P \in \operatorname{spec}(R) \mid \alpha(P)=0\}=$ $D(0: \alpha)$. Finally, a point $x$ in a topological space is said to be an $n$-point ( $n$ a positive integer) provided $x$ is in the closure of each of $n$ pairwise disjoint open sets.

Our main results on basic elements will be for (von Neumann) regular rings. In this context, the condition $M_{P} \neq 0$ for all $P$ ( $M$ finitely generated) simply says that $M$ is faithful. (Indeed, this is true over any reduced ring.) Also, since every localization of a regular ring is a field, an element $m \in M$ is basic at $P$ if and only if $m(P) \neq 0$.

LEMMA 4.1. Let $M$ be a finitely generated module over the regular ring $R$. Then $M$ has a basic element if (and only if) for every $x \in \operatorname{spec}(R)$ there is an element $m \in M$ such that $x \notin Z(m)^{-}$. 
Proof. The method of proof is entirely standard, and is implicit throughout [8]: Choose, for each $x \in \operatorname{spec}(R)$, an element $m_{x}$ such that $m_{x}(y) \neq 0$ for all $y$ in a neighborhood $U_{x}$ of $x$. Choose a finite disjoint refinement $\left\{V_{1}, \cdots, V_{n}\right\}$ of the open cover $\left\{U_{x}\right\}$, say $V_{i} \subseteq U_{x_{i} \cdot}$ Finally, choose idempotents $e_{i}$ such that $V_{i}=D\left(e_{i}\right)$, and set $b=$ $e_{1} x_{1}+\cdots+e_{n} x_{n}$. Then $b$ is the required basic element.

THEOREM 4.2. Let $R$ be a regular ring. If $\operatorname{spec}(R)$ has no 3points, then every two-generator faithful $R$-module has a basic element. The converse holds if $R$ is a Boolean ring.

Proof. Suppose $\operatorname{spec}(R)$ has no 3-points, and let $M=$ $R \alpha+R \beta$. Since $M$ is faithful, the open sets $Z(\alpha), Z(\beta), Z(\alpha+\beta)$ are pairwise disjoint. Hence, each point of $\operatorname{spec}(R)$ is outside the closure of at least one of these sets. By (4.1), $M$ has a basic element.

Conversely, assume $R$ is Boolean, and $z$ is in the closure of each of the pairwise disjoint open sets $D\left(A_{i}\right)$, where $A_{i}$ is an ideal of $R$, $i=1,2,3$. We consider the module constructed in the proof of $[8$, Proposition 20.1]. Let $T=\left\{\left(a_{1}+a_{3}, a_{2}+a_{3}\right) \in R \oplus R \mid a_{i} \in A_{i}\right\}$ and set $M=(R \oplus R) / T$. Let $\alpha_{1}$ and $\alpha_{2}$ be the images in $M$ of $(1,0)$ and $(0,1)$, respectively. It is easily seen that $A_{i}=\left(0: \alpha_{i}\right), i=1,2$, and $A_{3}=$ (0: $\left.\alpha_{1}+\alpha_{2}\right)$; that is, $D\left(A_{i}\right)=Z\left(\alpha_{i}\right)$ and $D\left(A_{3}\right)=Z\left(\alpha_{1}+\alpha_{2}\right)$.

Suppose $\alpha=e_{1} \alpha_{1}+e_{2} \alpha_{2}$ is a basic element of $M$. If, say, $e_{1}(z)=0$ and $e_{2}(z)=1$, then $N=Z\left(e_{1}\right) \cap Z\left(1-e_{2}\right)$ is a neighborhood of $z$, and hence meets $Z\left(\alpha_{2}\right)$. But $\alpha(x)=0$ for all $x \in N \cap Z\left(\alpha_{2}\right)$, a contradiction. The other two cases $\left(e_{1}(z)=1, e_{2}(z)=0 ; e_{1}(z)=e_{2}(z)=\right.$ 1) are handled similarly.

One can easily produce Boolean rings whose spectra contain 3-points. In fact, Pierce [8, Lemma 21.5] has shown that every infinite Boolean ring has a homomorphic image whose spectrum has a 3point. It follows that every infinite Boolean ring has a 2-generator module $M$ such that the annihilator of each element of $M$ is strictly larger than the annihilator of $M$.

It is unknown to the authors whether the converse of (4.2) holds in any regular ring. There seems to be no way of extending the proof given above for Boolean rings. For example, if none of the residue fields of $R$ has characteristic 2 , then $\alpha_{1}-\alpha_{2}$ is a basic element of $M$.

Can "two-generator" be replaced by "finitely-generated" in (4.2)? This seems plausible, but, again, no straight-forward extension of the method of proof of (4.2) seems to work.

A module $M$ over a regular ring is a generator of the category of $R$-modules if and only if $M$ has a unimodular element, [13, 4.2]. Carl Faith has asked whether being a generator is a local property for finitely generated modules. The next theorem gives a negative answer. 
THEOREM 4.3. A regular ring $R$ is self-injective if and only if finitely generated faithful $R$-module has a unimodular element.

Proof. Suppose $R$ is self-injective, and let $m_{1}, \cdots, m_{k}$ generate $M$. Then the map $R \rightarrow M^{k}$ taking $r$ to $\left(r m_{1}, \cdots, r m_{k}\right)$ is a split monomorphism. By the remark above, $M$ has a unimodular element.

Now suppose $R$ is not self-injective. There are two cases to consider. Case 1: $\operatorname{spec}(R)$ contains a 2-point. Say $P \in D(I)^{-} \cap$ $D(J)^{-}$where $I$ and $J$ are ideals of $R$ with $I \cap J=0$. Then, by [10, Proposition 2.6], $(0: I)+(0: J) \subseteq P$. Hence, by [10, Theorem 1.4], the faithful module $R / I \oplus R / J$ has no unimodular element. Case 2: $\operatorname{spec}(R)$ contains no 2-points. Let $M$ be the two-generator module $\Gamma(X, \mathscr{A})$, where $X=\operatorname{spec}(R)$ and $\mathscr{A}$ is the sheaf of modules constructed in [8, p. 107]. This module is nonsingular ("torsion-free" in the terminology of [8]), but not projective. Also, $M$ is faithful, by formula (1) of [8, p. 107]. Suppose, by way of contradiction, that $m$ is a unimodular element of $M$, that is, $M=R m \oplus M^{\prime}$. Then $M^{\prime}$ is locally cyclic, hence cyclic by (2.1), say $M^{\prime}=R / I$. Now, $M^{\prime}$ is nonsingular, so $D(I)$ is the interior of its own closure, by [8, Corollary 22.2]. But since $\operatorname{spec}(R)$ has no 2-points it follows that $D(I)$ is both open and closed, that is, $I$ is generated by an idempotent. Therefore $M$ is projective, a contradiction.

It is well-known that a Boolean ring $R$ is self-injective if and only if $\operatorname{spec}(R)$ has no 2-points. (See, for example, [8, Corollary 24.2].) thus for Boolean rings, we have the following parallel with (4.2):

Corollary 4.4. Let $R$ be a Boolean ring. Then $\operatorname{spec}(R)$ has no 2-points if and only if every two-generator faithful $R$-module has a unimodular element.

In view of (4.2) and (4.4), it is worth remarking that there exist Boolean rings whose spectra have 2-points but no 3-points [8, pp. 92, 93].

Acknowledgment. The authors are grateful to Thomas S. Shores for several helpful conversations related to this research.

\section{REFERENCES}

1. P. M. Cohn, Free Rings and Their Relations, Academic Press, London, 1971.

2. O. Forster, Über die Anzahl der Erzeugenden eines Ideals in einem Noetherschen Ring, Math. Zeitschrift, 84 (1964), 80-87.

3. L. Gillman and M. Henriksen, Rings of continuous functions in which every finitely generated ideal is principal, Trans. Amer. Math. Soc., 82 (1956), 366-391.

4. M. Henriksen, Some remarks on elementary divisor rings II, Mich. Math. J., 3 (1955-56), 159-163. 
5. I. Kaplansky, Projective modules, Ann. of Math., (2), 68 (1958), 372-377.

6. I. Kaplansky, Commutative Rings, Allyn and Bacon, Boston (1970).

7. M. D. Larsen, W. J. Lewis and T. S. Shores, Elementary divisor rings and finitely presented modules, Trans. Amer. Math. Soc., 187 (1974), 231-248.

8. R. S. Pierce, Modules over commutative regular rings, Mem. Amer. Math. Soc., 70 (1967).

9. R. G. Swan, The number of generators of a module, Math. Zeitschrift, 102 (1967), 318-322.

10. T. S. Shores and R. A. Wiegand, Rings whose finitely generated modules are direct sums of cyclics, J. Algebra, 32 (1974), 152-172.

11. W. Vasconcelos, Projective modules of finite rank, Proc. Amer. Math. Soc., 22, No. 2 (1969), 430-433.

12. R. A. Wiegand, Modules over universal regular rings, Pacific J. Math., 39, No. 3 (1971), 807-819.

13. R. A. Wiegand, Generators of modules over commutative rings, J. Algebra, 27, No. 3 (1973), $454-461$.

Received January 23, 1974 and in revised form April 16, 1974. The first author's research was supported by NSF Grant GP-37823.

UNIVERSITY OF NEBRASKA 


\section{PACIFIC JOURNAL OF MATHEMATICS}

\section{EDITORS}

RICHARD ARENS (Managing Editor)

University of California

Los Angeles, California 90024

\section{J. DugundJI}

Department of Mathematics University of Southern California Los Angeles, California 90007

D. Gilbarg and J. Milgram

Stanford University

Stanford, California 94305

\section{ASSOCIATE EDITORS}
E. F. BECKENBACH
B. H. NeumanN
F. WoLF
K. YoshiDA

\section{SUPPORTING INSTITUTIONS}

\author{
UNIVERSITY OF BRITISH COLUMBIA \\ CALIFORNIA INSTITUTE OF TECHNOLOGY \\ UNIVERSITY OF CALIFORNIA \\ MONTANA STATE UNIVERSITY \\ UNIVERSITY OF NEVADA \\ NEW MEXICO STATE UNIVERSITY \\ OREGON STATE UNIVERSITY \\ UNIVERSITY OF OREGON \\ OSAKA UNIVERSITY
}

\author{
UNIVERSITY OF SOUTHERN CALIFORNIA \\ STANFORD UNIVERSITY \\ UNIVERSITY OF TOKYO \\ UNIVERSITY OF UTAH \\ WASHINGTON STATE UNIVERSITY \\ UNIVERSITY OF WASHINGTON \\ AMERICAN MATHEMATICAL SOCIETY
}

The Supporting Institutions listed above contribute to the cost of publication of this Journal, but they are not owners or publishers and have no responsibility for its contents or policies.

Mathematical papers intended for publication in the Pacific Journal of Mathematics should be in typed form or offset-reproduced (not dittoed), double spaced with large margins. Underline Greek letters in red, German in green, and script in blue. The first paragraph or two must be capable of being used separately as a synopsis of the entire paper. Items of the bibliography should not be cited there unless absolutely necessary, in which case they must be identified by author and Journal, rather than by item number. Manuscripts, in duplicate, may be sent to any one of the four editors. Please classify according to the scheme of Math. Reviews, Index to Vol. 39. All other communications should be addressed to the managing editor, or Elaine Barth, University of California, Los Angeles, California, 90024.

100 reprints are provided free for each article, only if page charges have been substantially paid. Additional copies may be obtained at cost in multiples of 50 .

The Pacific Journal of Mathematics is issued monthly as of January 1966. Regular subscription rate: $\$ 72.00$ a year (6 Vols., 12 issues). Special rate: $\$ 36.00$ a year to individual members of supporting institutions.

Subscriptions, orders for back numbers, and changes of address should be sent to Pacific Journal of Mathematics, 103 Highland Boulevard, Berkeley, California, 94708.

PUBLISHED BY PACIFIC JOURNAL OF MATHEMATICS, A NON-PROFIT CORPORATION Printed at Jerusalem Academic Press, POB 2390, Jerusalem, Israel.

$$
\begin{gathered}
\text { Copyright (C) } 1975 \text { Pacific Journal of Mathematics } \\
\text { All Rights Reserved }
\end{gathered}
$$




\section{Pacific Journal of Mathematics}

\section{Vol. 58, No. $2 \quad$ April, 1975}

Zvi Artstein and John Allen Burns, Integration of compact set-valued functions . . . . . . . . . 297

Mark Benard, Characters and Schur indices of the unitary reflection group $[321]^{3} \ldots \ldots \ldots . .309$

Simeon M. Berman, A new characterization of characteristic functions of absolutely continuous

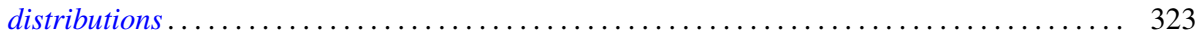

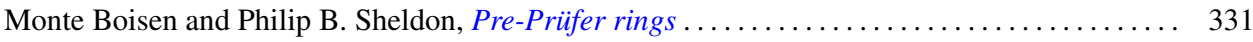

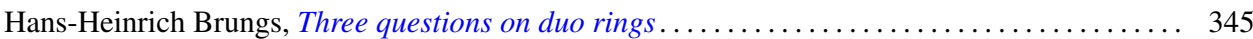

Iracema M. Bund, Birnbaum-Orlicz spaces of functions on groups................. 351

John D. Elwin and Donald R. Short, Branched immersions between 2-manifolds of higher

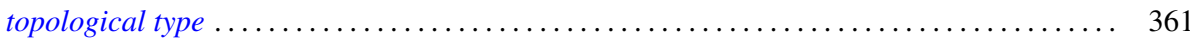

Eric Friedlander, Extension functions for rank 2, torsion free abelian groups . .......... 371

Jon Froemke and Robert Willis Quackenbush, The spectrum of an equational class of

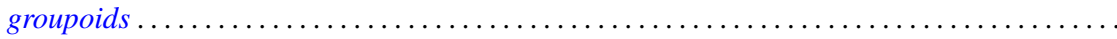

Barry J. Gardner, Radicals of supplementary semilattice sums of associative rings ...........

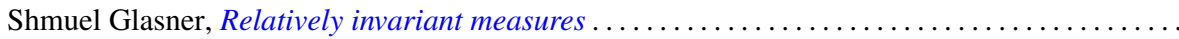

George Rudolph Gordh, Jr. and Sibe Mardesic, Characterizing local connectedness in inverse

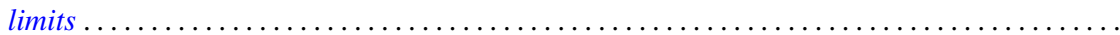

Siegfried Graf, On the existence of strong liftings in second countable topological spaces......

Stanley P. Gudder and D. Strawther, Orthogonally additive and orthogonally increasing

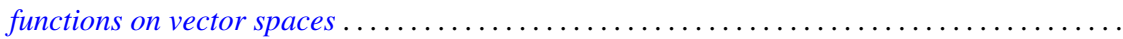

Darald Joe Hartfiel and Carlton James Maxson, A characterization of the maximal monoids and

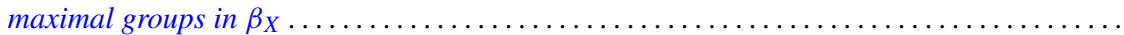

Robert E. Hartwig and S. Brent Morris, The universal flip matrix and the generalized faro-shuffle. .

William Emery Haver, Mappings between ANRs that are fine homotopy equivalences. .

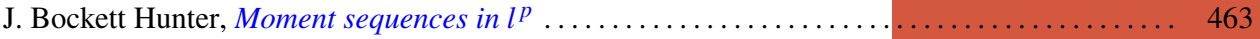

Barbara Jeffcott and William Thomas Spears, Semimodularity in the completion of a poset.... 467

Jerry Alan Johnson, A note on Banach spaces of Lipschitz functions . . . . . . . . . . . . 475

David W. Jonah and Bertram Manuel Schreiber, Transitive affine transformations on

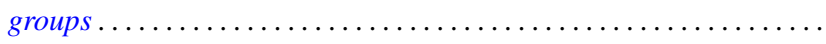

Karsten Juul, Some three-point subset properties connected with Menger's characterization of

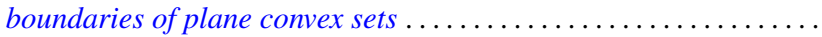

Ronald Brian Kirk, The Haar integral via non-standard analysis . . . . . . . . . . . . . 517

Justin Thomas Lloyd and William Smiley, On the group of permutations with countable

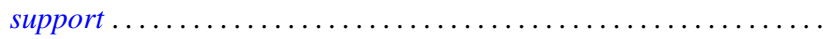

Erwin Lutwak, Dual mixed volumes .................................. 531

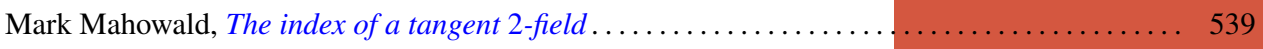

Keith Miller, Logarithmic convexity results for holomorphic semigroups . . . . . . . . . . . . 549

Paul Milnes, Extension of continuous functions on topological semigroups . . . . . . . . . . 553

Kenneth Clayton Pietz, Cauchy transforms and characteristic functions ................ 563

James Ted Rogers Jr., Whitney continua in the hyperspace $C(X) \ldots \ldots \ldots \ldots \ldots \ldots \ldots \ldots .569$

Jean-Marie G. Rolin, The inverse of a continuous additive functional . . . . . . . . . . . . 585

William Henry Ruckle, Absolutely divergent series and isomorphism of subspaces . ........ 605

Rolf Schneider, A measure of convexity for compact sets . ..................... 617

Alan Henry Schoenfeld, Continous measure-preserving maps onto Peano spaces .......... 627

V. Merriline Smith, Strongly superficial elements .......................... 643

Roger P. Ware, A note on quadratic forms over Pythagorean fields . . . . . . . . . . . . . . 651

Roger Allen Wiegand and Sylvia Wiegand, Finitely generated modules over Bezout rings . . . . 655

Martin Ziegler, A counterexample in the theory of definable automorphisms . . . . . . . . . 665 